\title{
Retrospective study of threshold time for the conventional treatment of branch retinal artery occlusion
}

This article was published in the following Dove Press journal:

Clinical Ophthalmology

22 September 2014

Number of times this article has been viewed

\author{
Daisuke Muramatsu* \\ Teruumi Minezaki* \\ Kinya Tsubota* \\ Yoshihiro Wakabayashi \\ Hiroshi Goto \\ Department of Ophthalmology, Tokyo \\ Medical University, Tokyo, Japan \\ *These authors contributed equally \\ to this work
}

Purpose: To investigate the medical backgrounds of patients and the treatment periods from the onset of branch retinal artery occlusion to obtaining improved final visual acuity.

Methods: This was a retrospective case series study. A total of 68 consecutive patients (69 eyes) with branch retinal artery occlusion who visited Tokyo Medical University Hospital from 2007 to 2012 were included in this study. All patients underwent ophthalmic examinations and visual acuity tests. We reviewed their medical records for systemic conditions, as well as the periods from onset of symptoms to treatment. Participants were categorized into 2 groups: group A ( $n=36)$, which received any treatment within 24 hours from onset, and group $B(n=33)$, which visited our hospital after 24 hours from onset. Best corrected visual acuity (BCVA) changes from the first to final visit and the relationships between systemic condition and visiting time to BCVA were assessed.

Results: At the first visit, 59\% of the patients had BCVA over 20/40; the ratio was increased to $74 \%$ at the final visit. BCVA improved more than 2 lines for $35 \%$ of the patients and was unchanged for $57 \%$ of those receiving conventional treatment. BCVA over 20/40 was significantly lower in hyperlipidemia patients. Hypertension, diabetes mellitus, and significant carotid stenosis were not correlated. The mean BCVA at baseline $(0.91 \pm 1.03)$ significantly recovered to $0.35 \pm 0.91$ after treatment in group A $(P<0.001$, Student's $t$-test $)$. The mean BCVA at baseline $(0.30 \pm 0.64)$ was $0.25 \pm 0.61$ at the final visit in group B (no significant change).

Conclusion: Conventional treatment within 24 hours from onset was acceptable for branch retinal artery occlusion.

Keywords: branch retinal artery occlusion, BRAO, golden period, treatment time

\section{Introduction}

The natural course of branch retinal artery occlusion (BRAO) is relatively favorable..$^{1-3}$ In contrast, the visual prognosis in central retinal artery occlusion (CRAO), although it is a disease similar to BRAO, is very poor. It is well known that the golden period for the treatment of CRAO (ie, as soon as possible from the onset of CRAO) is very important. ${ }^{2,4,5}$ Information about the period from the onset of BRAO to treatment is lacking, however, and the threshold time to react to treatment is still unknown. Some reports suggest there is no need for aggressive treatments for BRAO. However, the prognostic factors are uncertain. The purpose of this study was to assess the treatment periods from the onset of BRAO and the medical backgrounds of patients to obtain improved final visual acuity (VA).

\section{Material and methods}

This retrospective case study reviewed 69 eyes of 68 patients with BRAO who visited Tokyo Medical University Hospital from 2007 to 2012. The study was approved by
Correspondence: Daisuke Muramatsu Department of Ophthalmology, Tokyo Medical University, 6-7-I Nishishinjuku, Shinjuku-ku, Tokyo 160-0023, Japan $\mathrm{Tel}+8 \mathrm{I} 3334261$ II

Email gmura0815@yahoo.co.jp 
the institutional review board of the hospital and adhered to the tenets of the Declaration of Helsinki. Written informed consent was obtained from all patients. The patients were

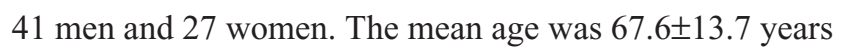
(range, 33-90 years). The targeted diseases included 46 eyes with BRAO, 15 eyes with hemi-CRAO, and eight eyes with cilioretinal artery occlusion (CLRAO). The study method was a retrospective case series based on medical records. The outcomes were best corrected visual acuity (BCVA; $\log$ MAR) at the first and final visits, the recovery ratio of BCVA, systemic medical condition, treatment methods, and period from onset to treatment.

\section{Treatment periods from onset}

All patients were categorized into 2 groups. Group A consisted of patients who received any treatment within 24 hours from onset. Group B consisted of patients who visited our hospital after 24 hours from onset. BCVA outcome and treatment method were assessed for these groups.

\section{Statistical analysis}

Statistical analysis was performed using StatView (Abacus Concepts Inc., Berkeley, CA, USA). The chi-square test was performed, testing the differences among occlusion type, systemic medical conditions, and BCVA recovery ratio. The Student's $t$-test was performed to compare the BCVA before and after treatment. A $P$-value of less than 0.05 was considered to indicate a statistically significant difference.

\section{Results}

The ratio of BCVA over 20/40 was 59\% (41 eyes) at the first visit; the ratio increased to $74 \%$ (54 eyes) at the final visit. The recovered eye ratio of BCVA over $0.2 \log$ MAR from the first to final visit improved in 24 eyes (35\%), was unchanged in 39 eyes (57\%), and worsened in six eyes ( $9 \%$ ).

We checked the relationships between final BCVA and systemic conditions, such as hypertension, hyperlipidemia, diabetes mellitus, and significant carotid stenosis. The ratio of final BCVA over 20/40 was significantly lower in patients with hyperlipidemia than in those without hyperlipidemia. However, there were no differences in improved BCVA in relation to hypertension, diabetes mellitus, and significant carotid stenosis (Table 1). With the assessment of occlusion type and final BCVA, there were no significant differences among BRAO, hemi-CRAO, and CLRAO (chi-square test). The occlusion was located from optic disc to the first artery-vessel crossing in 62 eyes. In seven eyes, the occlusion was located from the first arteryvessel crossing to peripheral. There were no significant differences between occlusion area and final BCVA (chisquare test). The interval from the onset of symptoms to treatment varied from 0.5 to 504 hours. The median time was 24 hours for all patients.

The number of eyes were 36 for group A (treated within 24 hours) and 33 for group B (visited hospital after 24 hours from onset). The mean age of the patients for each group was 65.6 \pm 15.4 for group A and 70.1 \pm 11.2 for group B. Group A had 22 men and 14 women, and group B had 20 men and 13 women. The past history for systemic conditions included 17 patients with hypertension, two with diabetes mellitus, ten with hyperlipidemia, and 20 with significant carotid stenosis in group A and 21 patients with hypertension, six with diabetes mellitus, eleven with hyperlipidemia, and 16 with significant carotid stenosis in group B. There were no significant differences between the two groups with respect to systemic conditions (chi-square test).

Table I Relationships between best corrected visual acuity and systemic medical conditions

\begin{tabular}{|c|c|c|c|}
\hline Systemic conditions & Number & $\begin{array}{l}\text { Ratio of best corrected } \\
\text { visual acuity over } 20 / 40\end{array}$ & $P$-value \\
\hline Hypertension & & & 0.867 \\
\hline Positive & 39 & $74 \%$ (27 eyes) & \\
\hline Negative & 17 & $76 \%$ (13 eyes) & \\
\hline Hyperlipidemia & & & 0.0143 \\
\hline Positive & 21 & $57 \%$ ( 12 eyes) & \\
\hline Negative & 20 & $90 \%$ ( 18 eyes) & \\
\hline Diabetes mellitus & & & 0.44 \\
\hline Positive & 8 & $63 \%$ (5 eyes) & \\
\hline Negative & 45 & $76 \%$ (34 eyes) & \\
\hline Significant carotid stenosis & & & 0.402 \\
\hline Positive & 18 & $61 \%$ (II eyes) & \\
\hline Negative & 26 & $73 \%$ ( 19 eyes) & \\
\hline
\end{tabular}


Table 2 Treatments

\begin{tabular}{lll}
\hline Treatment & Group A (within 24 hours; $\mathbf{n}=\mathbf{3 6})$ & Group B (more than 24 hours; $\mathbf{n}=\mathbf{3 3})$ \\
\hline Ocular massage & 36 eyes $(100 \%)$ & 31 eyes $(93 \%)$ \\
Paracentesis & 15 eyes $(41 \%)$ & 5 eyes $(13 \%)$ \\
Intraocular reduction by drug & 7 eyes $(19 \%)$ & 2 eyes $(8 \%)$ \\
Anticoagulant therapy & 25 eyes $(69 \%)$ & 19 eyes $(58 \%)$ \\
No treatment & 0 eyes $(0 \%)$ & 2 eyes $(8 \%)$ \\
\hline
\end{tabular}

Note: Some patients received overlapping treatments.

The treatment methods in group A were ocular massage for 36 eyes, paracentesis for 15 eyes, intraocular pressure reduction by drug for 7 eyes, and oral anticoagulant therapy for 25 eyes. All patients in group A received some treatment. In group B, ocular massage was performed for 31 eyes, paracentesis for five eyes, intraocular pressure reduction by drug for two eyes, and oral anticoagulant therapy for 19 eyes, and two patients received no treatment. In this study, there were no patients who received any systemic or local embolysis therapy in either group (Table 2).

The outcomes of BCVA change before and after treatment were as follows: the mean BCVA at baseline $(0.91 \pm 1.03)$ significantly recovered to $0.35 \pm 0.91$ after treatment in group $\mathrm{A}$ $(P<0.001$, Student's $t$-test; Figure 1A). However, the mean BCVA change before and after treatment in group B showed no statistically significant difference between $0.30 \pm 0.64$ at the first visit and $0.25 \pm 0.61$ at the final visit $(P=0.35$, Student's $t$-test; Figure 1B). BCVA improved (more than $0.2 \log M A R$ ) before and after treatment in $58 \%$ of the patients in group A and in $12 \%$ of the patients in group B (Figure 2). However, the ratio of BCVA over 20/40 at the first visit was higher in group B than in group A: $44.4 \%$ of the patients in group A had BCVA over 20/40 at the first visit compared with $75.8 \%$ of the patients in group B. Subgroup analysis was performed only for the poor BCVA cases with the exception of those who had BCVA over 20/40 at the first visit. In group A, the BCVA improved (more than 0.2 logMAR) in 15 eyes ( $75 \%$ ), was unchanged in four eyes (20\%), and decreased in one eye (5\%). In group B, the BCVA improved (more than 0.2 logMAR) in three eyes (37.5\%), was unchanged in three eyes $(37.5 \%)$, and decreased in two eyes (25\%). The improvement ratio was significantly higher in group $A$ than in group $B$ in these under 20/40 cases (chi-square test, $P=0.03$ ).

\section{Discussion}

Visual outcome after BRAO was relatively favorable. Several authors reported that $80 \%$ of BRAO patients could retain final VA over 20/40 without any aggressive treatment. ${ }^{1,2}$ In our study, most patients received some conventional therapy, although hemi-CRAO and CLRAO patients were also included. Nevertheless, $74 \%$ of the patients retained final BCVA over 20/40. We evaluated the relationship between favorable final BCVA to occlusion type, finding no differences between occlusion types (BRAO in the narrow sense,
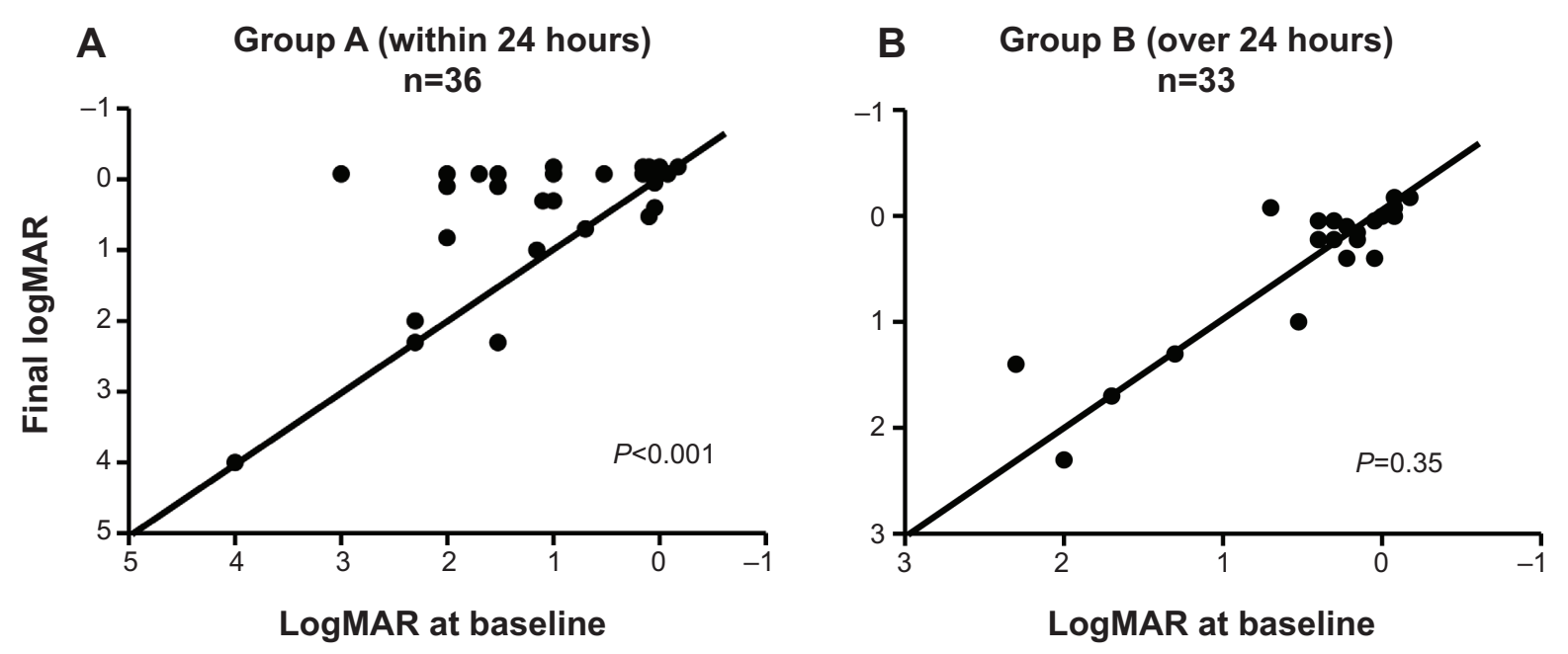

Figure I Best corrected visual acuity before and after treatment.

Notes: (A) Mean best corrected visual acuity at baseline $(0.9 \mathrm{I} \pm \mathrm{I} .03)$ significantly recovered to $0.35 \pm 0.9 \mathrm{I}$ after treatment in group $\mathrm{A}(P<0.00 \mathrm{I}$, Student's $t$-test). (B) In contrast, the mean best corrected visual acuity at baseline $(0.30 \pm 0.64)$ was not significantly different from the mean best corrected visual acuity at the final visit $(0.25 \pm 0.6 \mathrm{I})$ in group $B(P=0.35$, Student's $t$-test $)$. 


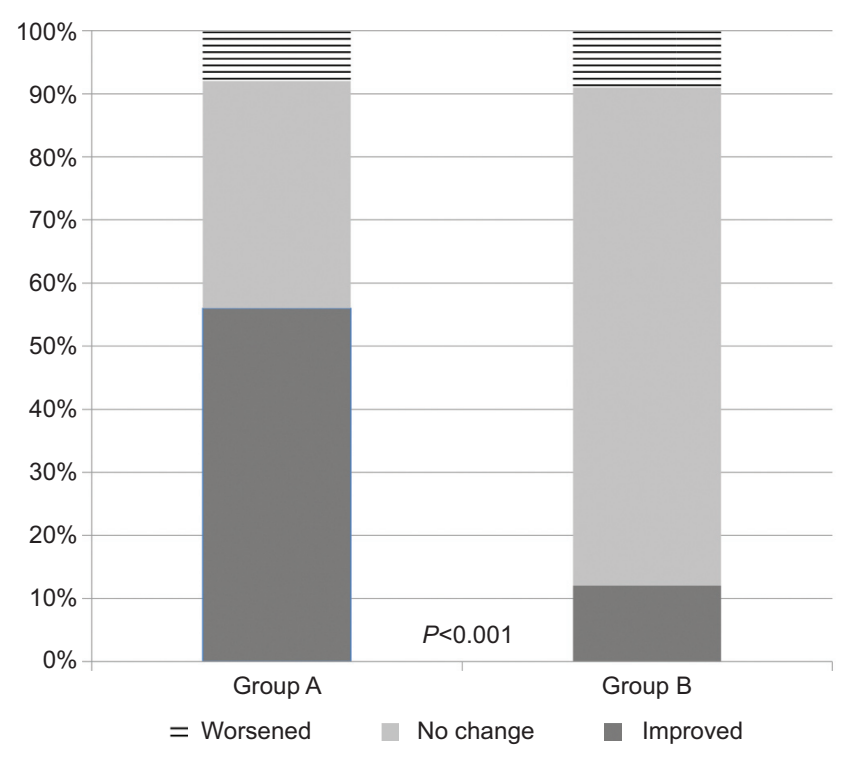

Figure 2 Ratio of best corrected visual acuity change ( $0.2 \log M A R)$ before and after treatment.

Notes: Best corrected visual acuity improved by more than 2 lines in $58 \%$ of patients in group $A$. The ratio of gain was significantly higher in the patients in group $A$ than in the patients in group $B(12 \%)$.

hemi-CRAO, and CLRAO) in relation to BCVA. Diabetes mellitus, hypertension, and infarction diseases are wellknown risk factors for BRAO. ${ }^{6}$ However, there were apparently no reports about the relationships between systemic medical conditions and VA in patients with BRAO.

In our study, many patients had past histories of systemic diseases, and an important finding was that hyperlipidemia was related to a poor prognosis of VA. The mechanisms of BRAO are of two types; namely, embolic occlusion and vasospasm. ${ }^{1}$ Hyperlipidemia patients have plaque in their blood vessels, which can more easily lead to more severe embolic occlusion than cases of vasospasm.

The visual prognosis in CRAO, with more severe vessel occlusion than in BRAO, was very poor. Treatment within a limited time from onset, known as the golden period, can achieve better VA. An experimental monkey model showed that the retina sustains irreversible damage when the central retinal artery has been obstructed for 105 minutes. ${ }^{4}$ Rumelt et al reported that $73 \%$ of CRAO patients acquired visual improvement with aggressive systemic treatments within 12 hours from the onset of symptoms. ${ }^{5}$ Another report also noted a correlation between a shorter duration of visual impairment and better prognosis for visual recovery. ${ }^{7}$ However, there is apparently no report about the golden period for BRAO treatment. Hayreh et al reported that $79 \%$ of patients acquired VA improvement within 7 days from onset, but only $43 \%$ of patients acquired VA improvement after 7 days from onset in the cases of initial VA of less than 20/40. ${ }^{1}$ Hence, early treatment appears to be as important in BRAO cases as it is in CRAO cases to avoid retinal damage after artery obstruction and ischemia. In our study, the median time from onset to treatment was 24 hours. We therefore assessed the visual prognosis within or after 24 hours. The BCVA was significantly improved before and after treatment for the patients who received any treatment within 24 hours from onset, but there was no difference in the patients who visited the hospital after 24 hours from onset. These results require some clarification. The initial VA of the "after 24 hours" group was relatively better than that of the "within 24 hours" group. The lack of initial visual impairment explains the delayed visit and the resulting inability to attain significant VA improvement.

We performed subgroup analysis only for poor initial VA under 20/40. These results revealed the same tendency for significant improvement for the within 24 hours group and the after 24 hours group. Many reports showed the time interval to visit the hospital as ranging from 2 to within 7 days from the onset of symptoms. ${ }^{1-3}$ In our study, some patients visited our hospital within 1-2 hours from onset. The median time of only 24 hours is much earlier than in other past reports. One reason for this shorter visiting time is the location of our facility, which is convenient to ground transportation. The Japanese health insurance system also facilitates visiting the hospital day or night. Yuzurihara and Iijima reported 23 cases of BRAO, of which $80 \%$ could gain final VA over $20 / 40$ without any special treatment. ${ }^{2}$ In their article, the mean visiting time was 1.9 days. Mason et al reported that only $60 \%$ of patients gained final BCVA over 20/40, with a median visiting time of 2.9 days. ${ }^{3}$ The time from the onset of symptoms to visiting the hospital is very important for VA recovery, preferably within 24-48 hours.

In our study, only conventional treatment was performed, and no patient received aggressive therapy such as systemic thrombolysis, counterpulsation, embolysis/embolectomy with a YAG laser, or local intra-arterial fibrinolysis by tissue plasminogen activator. ${ }^{5,8-10}$ Nevertheless, the final outcomes of VA were almost equal in these reports, including our study. We believe there is no need for aggressive treatment, which also has the benefit of avoiding possible severe adverse effects.

We conclude that $92 \%$ of the patients could obtain improved VA, and $75 \%$ of them had BCVA of over 20/40, with only conventional treatment within 24 hours from the onset of symptoms.

Study limitations included our study being a retrospective case series lacking a control group. Further studies are required to define the golden period and the best treatments. 


\section{Disclosure}

The authors report no conflicts of interest in this work.

\section{References}

1. Hayreh SS, Podhajsky PA, Zimmerman MB. Branch retinal artery occlusion: natural history of visual outcome. Ophthalmology. 2009;116(6): 1188-1194.e1, 4.

2. Yuzurihara D, Iijima H. Visual outcome in central retinal and branch retinal artery occlusion. Jpn J Ophthalmol. 2004;48(5):490-492.

3. Mason JO III, Shah AA, Vail RS, Nixon PA, Ready EL, Kimble JA. Branch retinal artery occlusion: visual prognosis. Am J Ophthalmol. 2008;146(3):455-457.

4. Hayreh SS, Weingeist TA. Experimental occlusion of the central artery of the retina. IV: retinal tolerance time to acute ischaemia. Br JOphthalmol. 1980;64(11):818-825.

5. Rumelt S, Dorenboim Y, Rehany U. Aggressive systematic treatment for central retinal artery occlusion. Am J Ophthalmol. 1999;128(6): $733-738$.
6. Hayreh SS, Podhajsky PA, Zimmerman MB. Retinal artery occlusion: associated systemic and ophthalmic abnormalities. Ophthalmology. 2009;116(10):1928-1936.

7. Augsburger JJ, Magargal LE. Visual prognosis following treatment of acute central retinal artery obstruction. Br J Ophthalmol. 1980; 64(12):913-917.

8. Werner D, Michalk F, Harazny J, Hugo C, Daniel WG, Michelson G. Accelerated reperfusion of poorly perfused retinal areas in central retinal artery occlusion and branch retinal artery occlusion after a short treatment with enhanced external counterpulsation. Retina. 2004; 24(4):541-547.

9. Opremcak E, Rehmar AJ, Ridenour CD, Borkowski LM, Kelley JK Restoration of retinal blood flow via translumenal Nd: YAG embolysis/ embolectomy (TYL/E) for central and branch retinal artery occlusion. Retina. 2008;28(2):226-235.

10. Schumacher M, Schmidt D, Jurklies B, et al; EAGLE-Study Group. Central retinal artery occlusion: local intra-arterial fibrinolysis versus conservative treatment, a multicenter randomized trial. Ophthalmology. 2010;117(7):1367-1375.e1.
Clinical Ophthalmology

\section{Publish your work in this journal}

Clinical Ophthalmology is an international, peer-reviewed journa covering all subspecialties within ophthalmology. Key topics include: Optometry; Visual science; Pharmacology and drug therapy in eye diseases; Basic Sciences; Primary and Secondary eye care; Patien Safety and Quality of Care Improvements. This journal is indexed on

Submit your manuscript here: http://www.dovepress.com/clinical-ophthalmology-journal

\section{Dovepress}

PubMed Central and CAS, and is the official journal of The Society of Clinical Ophthalmology (SCO). The manuscript management system is completely online and includes a very quick and fair peer-review system, which is all easy to use. Visit http://www.dovepress.com/ testimonials.php to read real quotes from published authors. 